\title{
A study on the individual pump flow estimation processor for optimal operation
}

\author{
Md Rakibuzzaman ${ }^{1}$, Sang-Ho Suh $^{2,3 *}$, In-Sik Yoon ${ }^{4}$, and Sang Yong Jung ${ }^{4}$ \\ ${ }^{1}$ Department of Mechanical Engineering, IUBAT-International University of Business Agriculture and \\ Technology, Dhaka, 1230, Bangladesh \\ ${ }^{2}$ Future Technology Institute, Anyang-si, Gyeonggi-Do, 14051, Korea \\ ${ }^{3}$ School of Mechanical Engineering, Soongsil University, Seoul, 06978, Korea \\ ${ }^{4}$ Dooch Co. Ltd., R \& D Center, 162 LS-ro, Gunpo-si, Gyeonggi-do, 15807, Korea
}

\begin{abstract}
The booster pump is an energy-saving system that controls the number of turns of the pump and optimal operation by installing an inverter to control the discharge pressure of the pump in accordance with the flow rate. The development of an optimum operation algorithm and flow rate information for the individual pumps without flow sensors is critical of a smart booster pump system. The objectives of this study was to investigate the algorithm based flow detection method without use of a flow sensor for each pump. Therefore, it was developed an algorithm-based flow rate detection method of individual pumps without the use of a flow sensor that has not been studied in the conventional booster pump system. The softwarebased processor development and experimental verification for detecting the flow rate of individual pumps were confirmed. The ensemble error was within $2 \%$. For optimal operation, the result of examining the accumulated flow rate for the sequential operation method and the optimum operation method. The flow measurement accuracy of the system was confirmed by experiment and the development of a booster pump system without a flow sensor was found effective.
\end{abstract}

\section{Introduction}

In large-scale apartments and commercial buildings, rapid response to flow rate changes is required. In most systems, multiple pumps are connected in parallel to deliver the required flow rate, and pressure and flow rate are measured as overall values for the booster pump. An inverter can be installed to drive the booster pump system (BPS) and control the rotation speed of the individual pumps. Currently, flow detection and energy savings method are the hot topic for BPSs [1]; however, few studies have investigated only on energy savings in BPSs both experimentally and numerically [2-6]. Rakibuzzaman et al. $(2015,2017)$ recently investigated on energy saving rates for a multistage, centrifugal pump with variable speed drive through numerically and experimentally [7], [8]. In order to improve the reliability of the booster pump, the optimum operation algorithm and flow rate information for the individual pumps of the booster pump system is required. The general booster pump system is controlled by using an integrated inverter and controller. By knowing the flow rate of each

\footnotetext{
Corresponding author: $\underline{\text { suhsh } @, \text { ssu.ac.kr }}$
} 
pump allows us to understand the operating conditions of individual pumps, which can be used to detect and maintain pump abnormalities. In the booster pump, if the master pump fails, other individual pumps automatically perform the function of the master pump. So, continuous water supply is possible and the durability of the pump can be improved. For the booster pumps, a pressure gauge is installed on the suction side and the discharge side of the pump system, so the head can be predicted, but it is not possible to measure the flow rate of each pump since only one flow meter is installed at the rear of the exit area. The device for measuring the flow rate by the hardware method is not only expensive but also there is a problem of the flowmeter error, and there is a concern of failure due to the substance. Recently, flow measurement accuracy has been studied and the accuracy of flow measurement in flow sensors has been discussed [9]. This paper focuses on the technology without the use of the flow sensor.

The objectives of this study are to the development of flow detection method without the use of a flow sensor that has never been studied in conventional booster pump systems and the development of a flow calculation processor for individual pumps in a booster pump system. Also, the optimum operation using flow rates readings was studied. In this study, the flow measurement accuracy of a developed flow control algorithm was evaluated.

\section{Methodology}

\subsection{Booster pump system}

Booster pump is a package system that combines a series of devices such as centrifugal pump, pressure tank, inverter, motor, pressure transmitter, suction/discharge conduit, control panel, base, etc. Booster pump operates by combining two or more centrifugal pumps in parallel. Figure 1 represents the booster pump system.
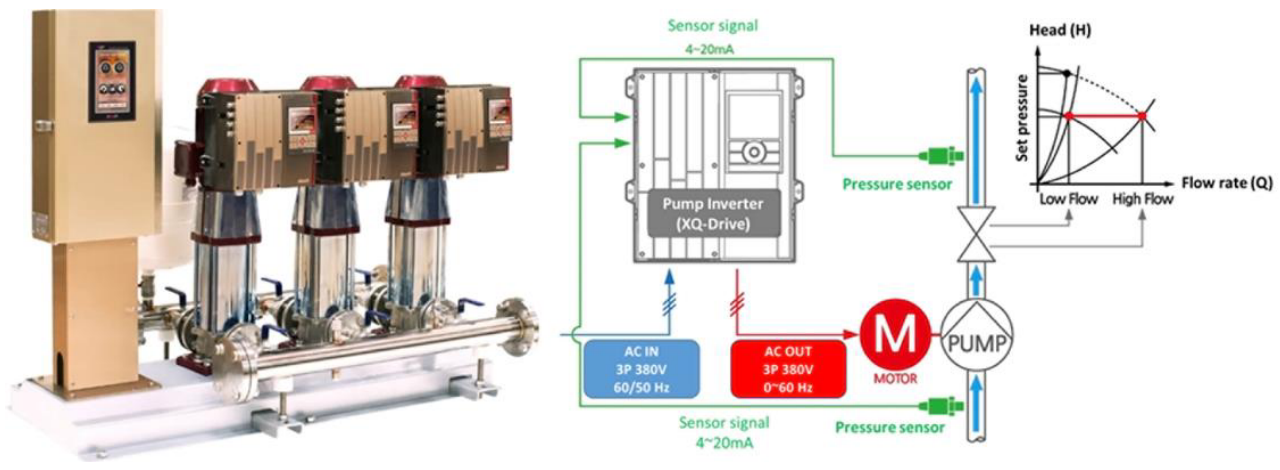

Fig. 1. Booster pump (IOP-3XRL15-3S) and its block diagram.

\subsection{Flow measurement method of individual pump}

For the booster pumps, a pressure gauge is installed on the suction and discharge side of each pump, so the head can be measured, but it is not possible to measure the flow rate of each pump since only one pump is installed at the rear of the exit pipe. For booster pumps that perform the optimum operation by controlling the inverter pressure (rotation speed change), flow rate information of each pump is required. There are two types of flow measurement methods; (1) hardware-based flow measurement and (2) software-based flow measurement method. In the hardware-based flow measurement method, a flow sensor is used to measure 
flow rates. The methodology for measuring the flow rate through this flow sensor was as follows: the turbine flow meter rotates as fluid flows through the check valve [10]. The flow rate sensor consists of a part of a hall sensor that detects movement of a rotating rotor, a part of a rotor, and an output part that processes and outputs electromotive force values generated by a hall sensor. The hall sensor part detects a change in current caused by magnetic fields inserted into the rotor when the rotating body rotates, the hall sensor represents the magnetic field as an electromotive force through the hall effect. Fig. 2 shows the check valve with a flow sensor. In the software-based flow control method, an algorithm was developed to estimate the flow rate by calculating the rotational speed and shaft power of the motor at the inverter and measuring the total head at the pump, and applying it to the flow rate-power curve and the flow rate-head curve of the pump.

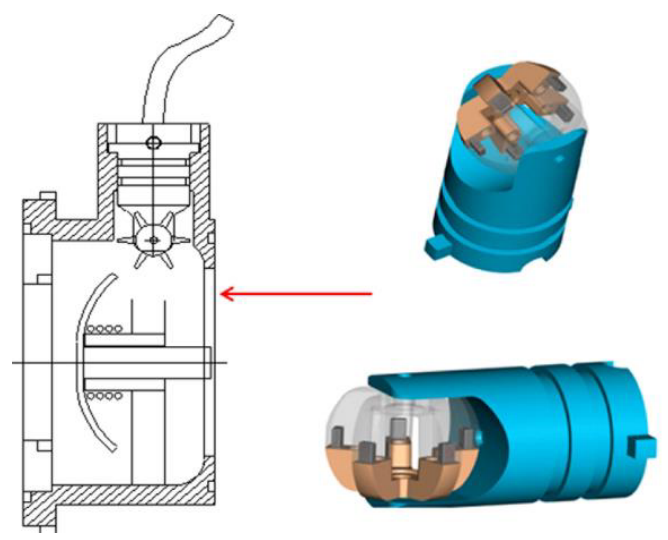

Fig. 2. Check valve with flow sensor in Booster pump system.

\subsection{Flow calculation processor for individual pumps}

The motor speed ( $n_{\text {est }}$ ) of an individual pump [11] is calculated using the Eq. 1:

$$
\begin{aligned}
& n_{\text {est }}=n_{\text {ref }}-n_{\text {slip }} \\
& n_{\text {slip }}=n_{\text {slip_rated }}\left(\frac{I_{\text {out }}-I_{\text {no_load }}}{I_{\text {rated }}-I_{\text {no_load }}}\right) \\
& I_{\text {out }}=\sqrt{i_{d s}^{2}-i_{q s}^{2}}
\end{aligned}
$$

where $n_{\text {ref }}$ is the inverter output speed, $n_{\text {slip }}$ is the motor slip speed, $n_{\text {slip_rated }}$ is the motor rated slip speed, $I_{\text {out }}$ is the motor output current, $I_{\text {rated }}$ is the rated current, and $I_{\text {no }}$ load is the no-load current. The estimation shaft output power of each pump is calculated using the Eq. 2:

$$
\begin{aligned}
& P_{e s t}=T_{e s t} \bullet n_{e s t} \\
& T_{e s t}=\frac{3}{2} \frac{P}{2} \frac{L_{m}^{2}}{L_{r}} i_{d s} i_{q s}
\end{aligned}
$$

where $T_{e s t}$ is the motor torque, $L_{m}$ is the motor mutual induction, $L_{r}$ is the motor rotor inductance. And the flow estimator provided in the inverter is calculated the total head of the 
pump from the measured pressure, flow velocity, and height at the suction and discharge measuring sections as [12]

$$
H_{\text {total }}=\frac{p_{\text {real }}-p_{\text {in }}}{\gamma}+\frac{V_{\text {real }}^{2}-V_{\text {in }}^{2}}{2 g}+\left(E_{\text {real }}-E_{\text {in }}\right)
$$

where $\gamma$ is the fluid specific weight, $p_{\text {real }}$ and $p_{\text {in }}$ are the pressure at discharge and suction of the pump, $V_{\text {real }}$ and $V_{\text {in }}$ are the average flow velocity at discharge and suction pressure sensor position, $E_{\text {real }}$ and $E_{i n}$ are the discharge and suction pressure sensor height.

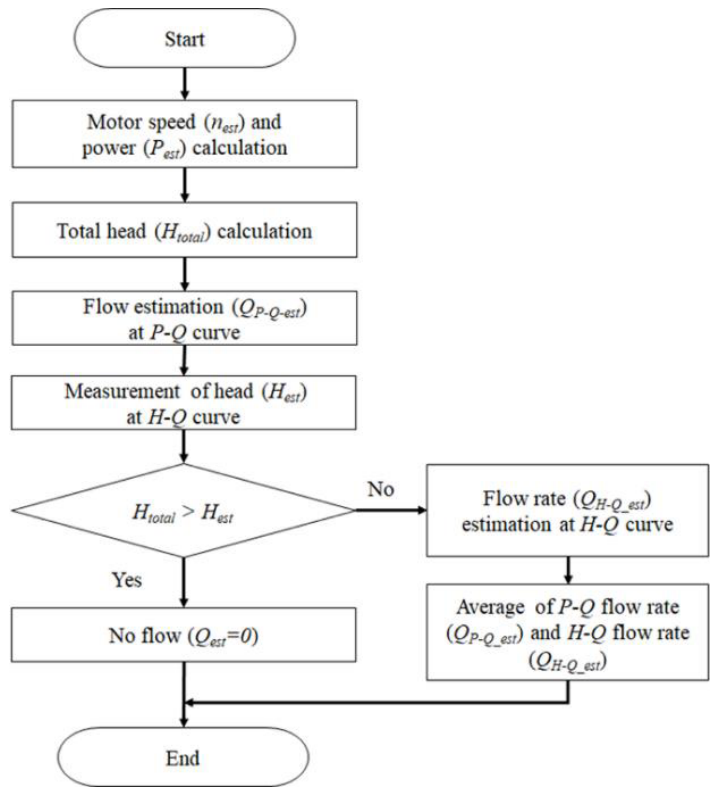

Fig. 3. Algorithm for calculating the flow rate of each individual pump.

Then an algorithm was developed for estimation of the flow rate. The algorithm configuration of the processor is shown in Fig. 3. In this system, firstly, find out the rotation speed and shaft power of the motor by the dedicated inverter and measure the total head in the pump. Then, the flow rate and the head are estimated using the $P-Q$ curve and the $H-Q$ curve. If the estimated head is smaller than the total head, there is no flow rate. If the estimated head is larger than the total head, calculate the flow rate using the $H-Q$ curve. Then calculate the average discharge flow rate from the $P-Q$ curve as shown in Fig. 4.
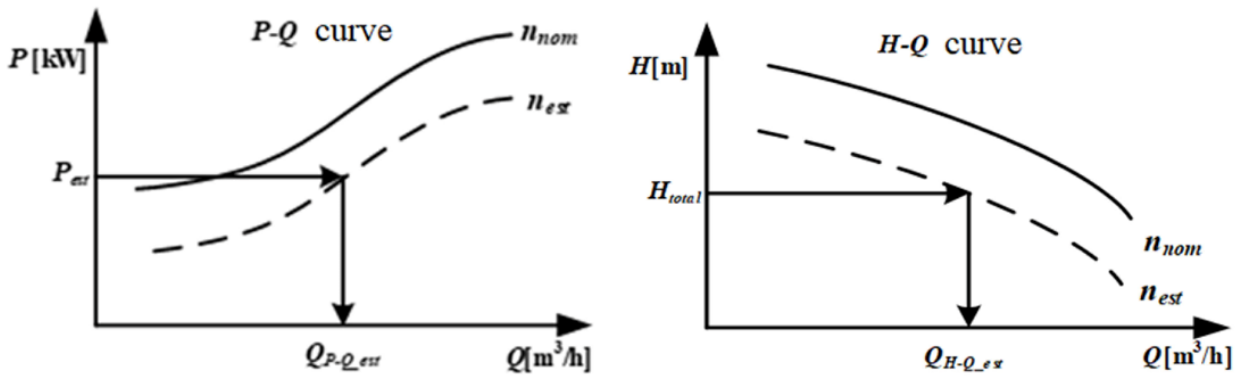

Fig. 4. Flow rate and head estimation method using P-Q curve and H-Q curve. 
This technology is commercialized by improving the algorithm for optimal operation control method using flow rate calculation of individual inverter booster pump system. It detects individual flow rates and calculates the accumulated flow rate for each pump and controls the operating priority, number of operations, and rotation speed of the pump based on this. The optimal operation algorithm starts in the order of $\mathrm{P}_{\mathrm{A}} \rightarrow \mathrm{P}_{\mathrm{B}} \rightarrow \mathrm{P}_{\mathrm{C}}$ and then controls the pump with the least accumulated flow to start first (e.g. $\mathrm{P}_{\mathrm{B}} \rightarrow \mathrm{P}_{\mathrm{C}} \rightarrow \mathrm{P}_{\mathrm{A}}$ ). When the number of pumps increases, the pump with the lowest accumulated flow rate of each pump is operated first. When the number of pumps decreases, the pump with the highest accumulated flow is stopped. In this case, it is necessary to have an optimal operation technology that switches the pump with the most accumulated power and the pump with the least. This technology can reduce power consumption by controlling the number of rotations, and can significantly improve the reliability of the booster pump. Figure 5 illustrates the optimal operation algorithm for each pump.

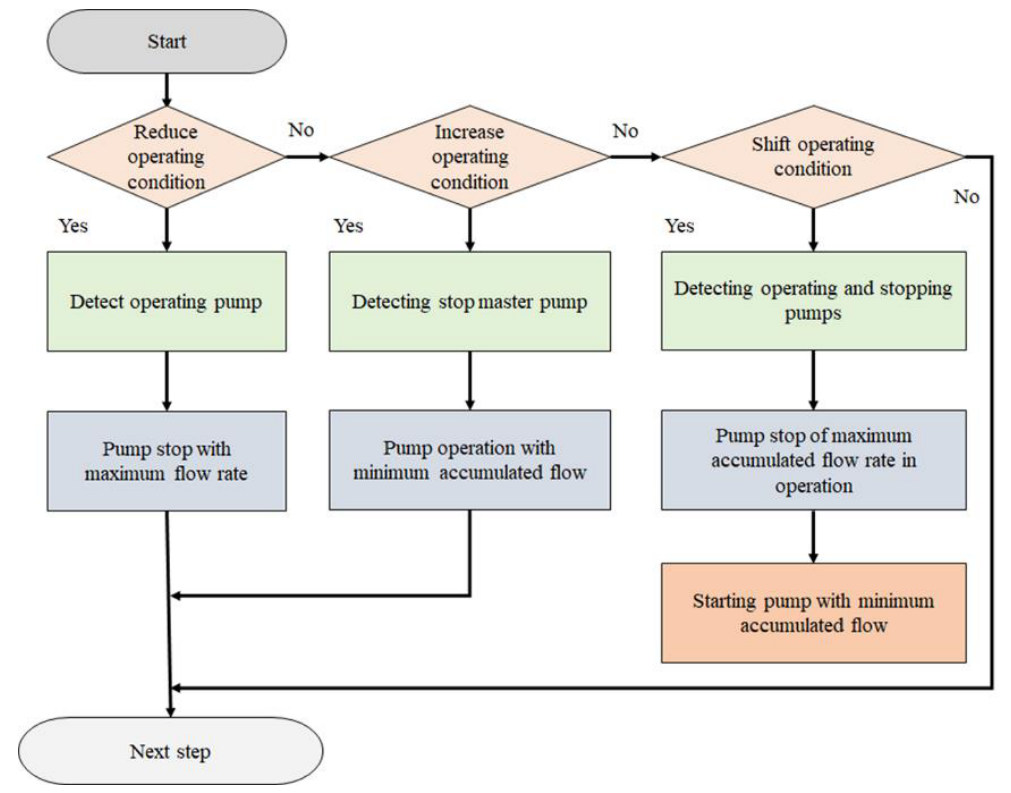

Fig. 5. Optimal operation algorithm.

\subsection{Experimental method}

The test facility was constructed according to KSB-6302 and ISO-5198 [13]. Flow measurement was performed at the Dooch test site as shown in Fig. 6. Three centrifugal pumps were installed with a reserve tank of $35 \mathrm{~m}^{3}$, and a pressure gauge and electromagnetic flowmeter were installed downstream of individual pumps. The pressure gauge ranges from 0 to 30 bar. The pressure tank operating range was $1.4-10$ bar. The inlet pipe diameter was $125 \mathrm{~mm}$ and the outlet pipe diameter was $100 \mathrm{~mm}$. Also, for measuring the estimated pump performance of the individual pumps, three digital VFD monitors were installed. This system was connected to a computer (PC) to obtain pressure, flow rate, and frequency without flow sensor readings. Experimental flow was measured with an electronic flow meter. Electronic flow meters were installed at the outlet of each pump and behind the outlet conduit. The total flow is measured by the electronic flow meter of the booster pump system. The test of the pump was, in principle, with clean water at a temperature $(0 \text { to } 40)^{\circ} \mathrm{C}$. However, the density at this time should be calculated. The standard density of water was $1000 \mathrm{~kg} / \mathrm{m}^{3}$. The test 
rotation speed was, in principle, AS-RUN speed. Here, the rotational speed generally means a speed converted into an inverter frequency. Set the required pressure to the inverter. The pressure sensor senses the pressure change in the pipe and sends it to the drive. The drive uses the set pressure and sense pressure to rotate the motor at the proper speed and control the discharge pressure of the pump.

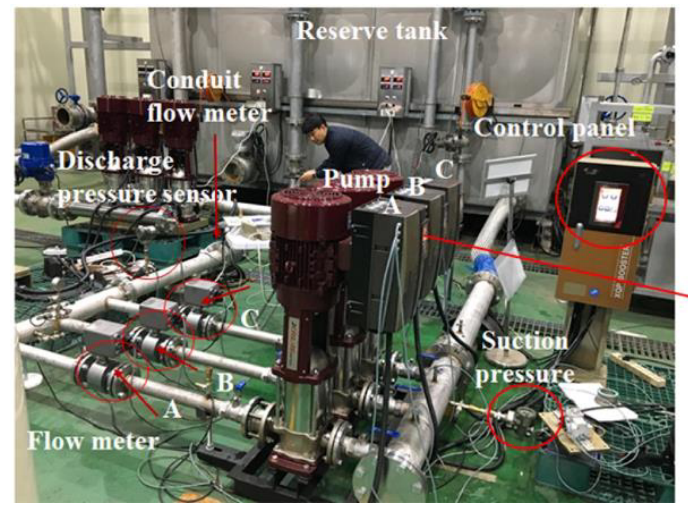

(a) Experimental devices of booster pump

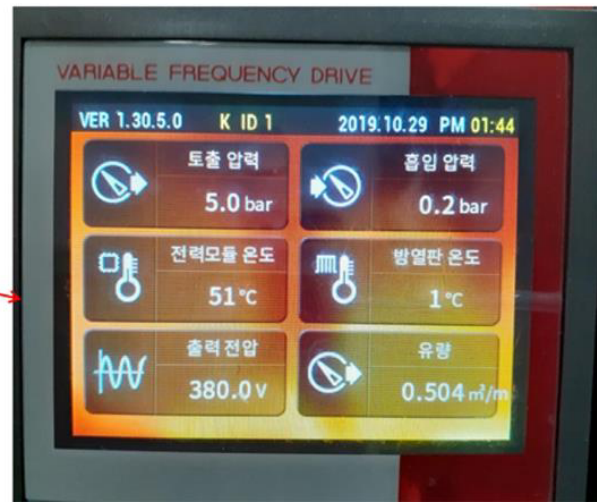

(b) Display panel of VFD

Fig. 6. Experimental facilities of the booster pump.

\section{Results and discussion}

\subsection{Performance characteristics of booster pump system}

In the experiment, three centrifugal pumps were installed and operated at the designed layout as pump $\mathrm{A}, \mathrm{B}$, and $\mathrm{C}$. To measure the performance of the individual pump, the suction pressure was set to $20 \mathrm{kPa}$ during one operation and the discharge flow rate was measured by changing the discharge pressure from 800 to $200 \mathrm{kPa}$ with $100 \mathrm{kPa}$ interval. Figure 7 shows the performance characteristics and \% of error of pump A. It is seen from the figure that the error rate between reference and estimation value of pump A was within $1 \%$. It was also confirmed that the error of the pump B and $\mathrm{C}$ lies within $3 \%$.

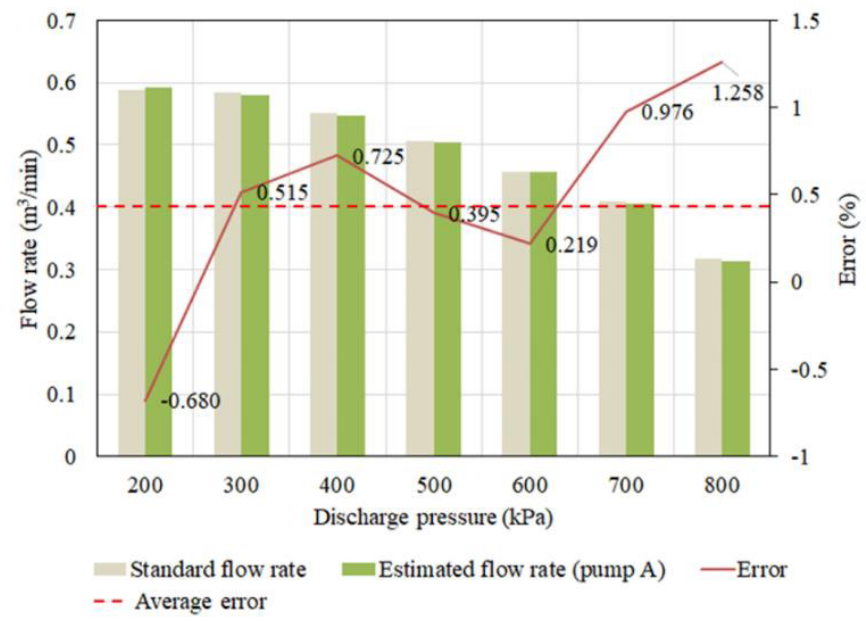

Fig. 7. Difference between estimated flow rate and actual flow rate of pump A. 


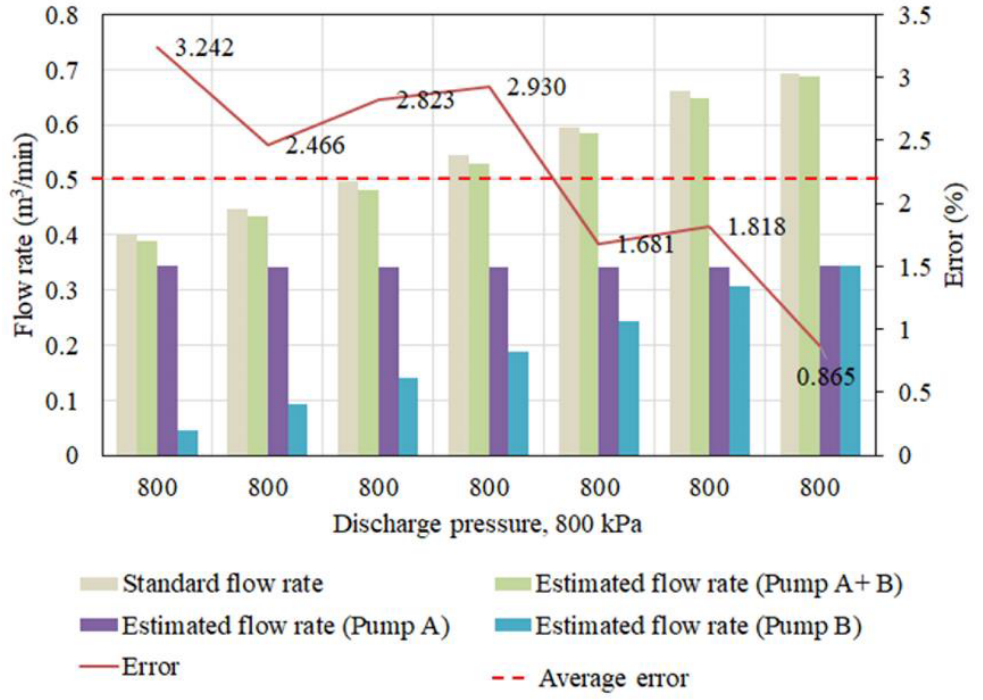

Fig. 8. Difference between estimated flow rate and actual flow rate during operation of two pumps.

For parallel operation in two pumps, the suction pressure was $20 \mathrm{kPa}$ and the discharge flow rate was measured at the discharge pressure of $800 \mathrm{kPa}$. Figure 8 illustrates the performance and error of two pumps in parallel. In this figure, it is observed that the error of the two pumps was within $1 \sim 3 \%$. For parallel operations in three pumps, the flow rate was measured at the discharge set pressure of $600 \mathrm{kPa}$. Figure 9 shows the performance comparison of reference and estimation of the three pumps in operations, and $\%$ of errors of three pumps. It is seen from the figure that the error rate of three pumps was less than $2 \%$. Table 1 shows the $\%$ of error of three pumps in parallel. Based on the experimental results, it was judged that using an algorithm-based flow detection method, the flow rate could be estimated without the use of a flow sensor.

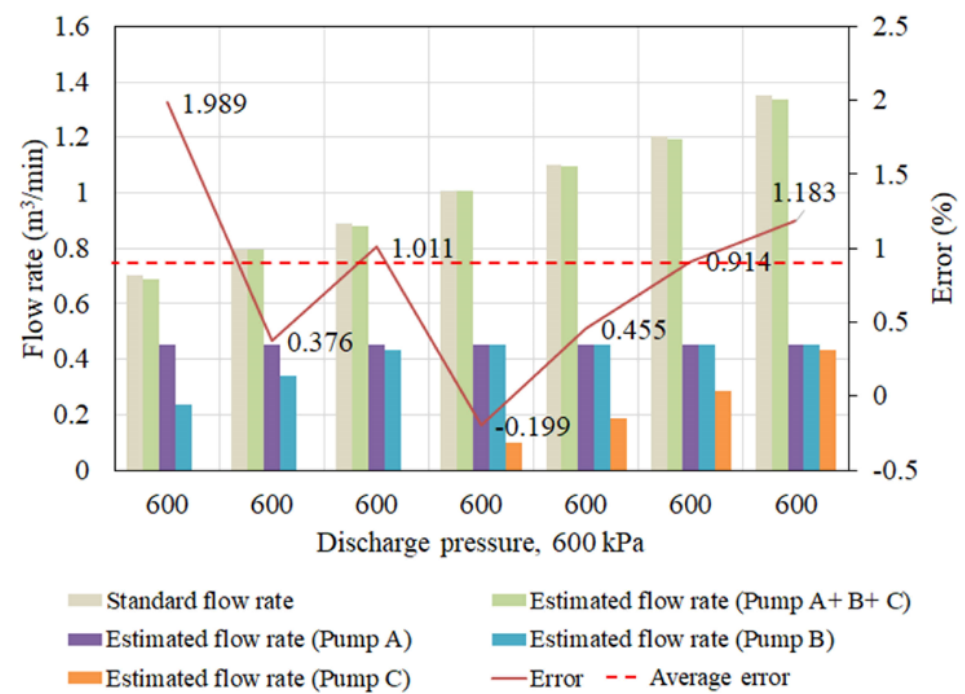

Fig. 9. Measurement, estimated flow rate and error of three pumps. 
Table 1. Pump experiment and estimation error of flow rate.

\begin{tabular}{|c|c|c|c|c|}
\hline \multicolumn{2}{|c|}{ Test items } & $\begin{array}{l}\text { Test results } \\
\left(\mathrm{m}^{3} / \mathrm{hr}\right)\end{array}$ & $\begin{array}{c}\text { Error } \\
(\%)\end{array}$ & Remarks \\
\hline \multirow{5}{*}{$\begin{array}{l}\text { Suction pressure: } 20 \mathrm{kPa} \\
\text { Discharge pressure: } 600 \\
\mathrm{kPa}\end{array}$} & Measured flow rate & 0.704 & \multirow{5}{*}{1.989} & \multirow{15}{*}{$\begin{array}{l}\text { Pump A: on } \\
\text { Pump B: on } \\
\text { Pump C: on }\end{array}$} \\
\hline & Estimated flow rate (Pump A) & 0.454 & & \\
\hline & Estimated flow rate (Pump B) & 0.236 & & \\
\hline & Estimated flow rate (Pump C) & 0.000 & & \\
\hline & Estimated flow rate & 0.690 & & \\
\hline \multirow{5}{*}{$\begin{array}{l}\text { Suction pressure: } 20 \mathrm{kPa} \\
\text { Discharge pressure: } 600 \\
\mathrm{kPa}\end{array}$} & Measured flow rate & 1.100 & \multirow{5}{*}{0.455} & \\
\hline & Estimated flow rate (Pump A) & 0.453 & & \\
\hline & Estimated flow rate (Pump B) & 0.453 & & \\
\hline & Estimated flow rate (Pump C) & 0.189 & & \\
\hline & Estimated flow rate & 1.095 & & \\
\hline \multirow{5}{*}{$\begin{array}{l}\text { Suction pressure: } 20 \mathrm{kPa} \\
\text { Discharge pressure: } 600 \\
\mathrm{kPa}\end{array}$} & Measured flow rate & 1.352 & \multirow{5}{*}{1.183} & \\
\hline & Estimated flow rate (Pump A) & 0.453 & & \\
\hline & Estimated flow rate (Pump B) & 0.452 & & \\
\hline & Estimated flow rate (Pump C) & 0.431 & & \\
\hline & Estimated flow rate & 1.336 & & \\
\hline
\end{tabular}

\subsection{Sequential and optimal operations method}

In the booster pump system, the conventional sequential operation method focuses on the accumulation flow rate of a particular pump, as shown in Fig. 10, while the optimal operation method of the booster pump was distributed evenly among individual pumps. For the flow pattern shown in Fig. 11, when the conventional sequential operation method and optimal operation method using the individual flow rate were operated, the excellence of the optimum equalization technology was verified by comparing the operation amount (accumulated flow rate) of the pump. The deviation of the experiment was calculated for each pump. Table 2 shows the comparison of flow rate between sequential and optimal operation methods. In this case, the suction pressure was set to $38 \mathrm{kPa}$ and discharge pressure was set to $700 \mathrm{kPa}$. From this table, it is observed that the average deviation for the conventional operational control method was higher than the developed operational control method of individual pumps.

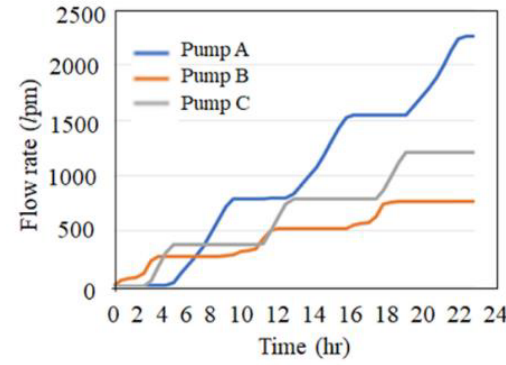

(a) Sequential operation

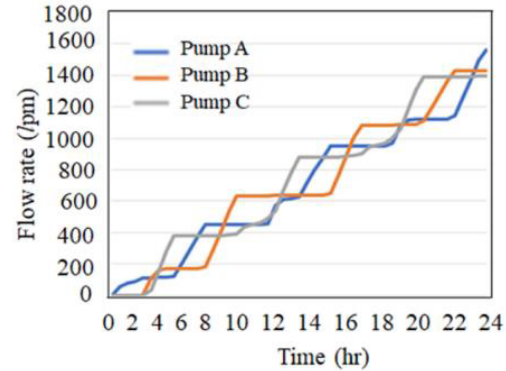

(b) Optimal operation

Fig. 10. Comparison of accumulated flow rate by pump between sequential operation method and application technology operation method. 


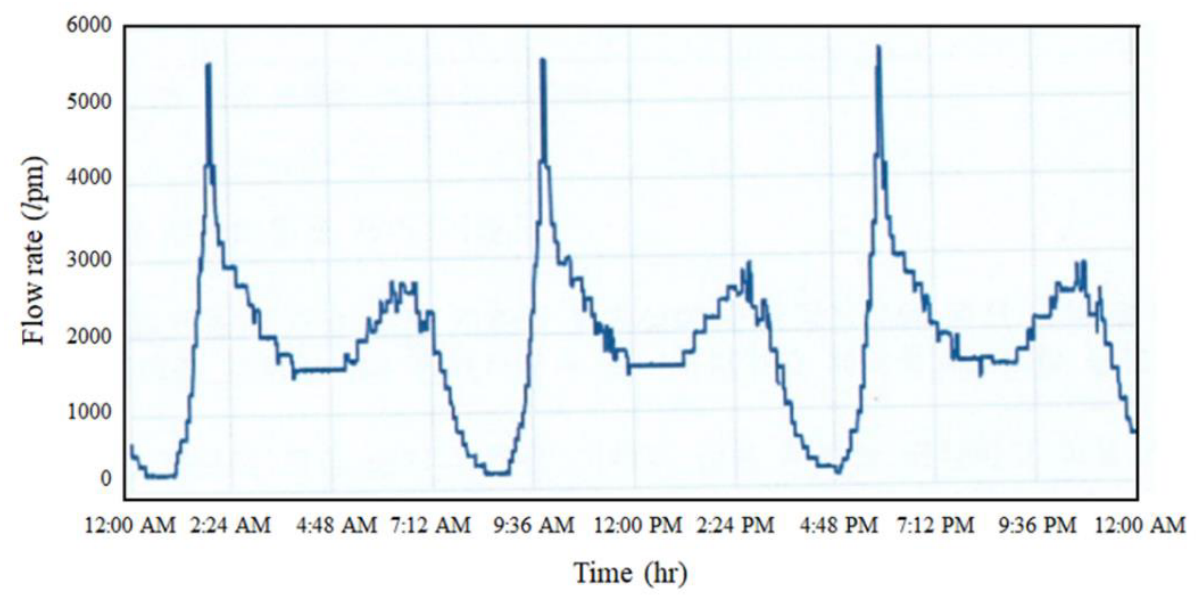

Fig. 11. Flow rate pattern for 24 hours (based on residential apartments).

Table 2. Standard deviation of individual pump.

\begin{tabular}{|l|l|c|c|}
\hline \multicolumn{2}{|c|}{} & \multicolumn{2}{c|}{ Experimental results } \\
\cline { 2 - 4 } \multicolumn{2}{|c|}{} & $\begin{array}{c}\text { Accumulated flow } \\
\text { rate }\left(\mathrm{m}^{3} / \text { day }\right)\end{array}$ & Deviation (\%) \\
\hline \multirow{3}{*}{$\begin{array}{l}\text { Sequential operation method } \\
\text { Suction pressure: } 38 \mathrm{kPa} \\
\text { Discharge pressure: } 700 \mathrm{kPa} \\
\text { Driving time: } 24 \mathrm{hr}\end{array}$} & Pump A & 226 & 59.9 \\
\cline { 2 - 4 } & Pump B & 76 & -46.2 \\
\cline { 2 - 4 } & Pump C & 122 & -13.7 \\
\cline { 2 - 4 } & Subtotal & 424 & - \\
\hline \multirow{2}{*}{$\begin{array}{l}\text { Optimal operation method } \\
\text { Discharge pressure: } 700 \mathrm{kPa} \\
\text { Driving time: } 24 \mathrm{hr}\end{array}$} & Pump A & 148 & -1.7 \\
\cline { 2 - 5 } & Pump B & 139 & -3.8 \\
\cline { 2 - 5 } & Pump C & 136 & - \\
\cline { 2 - 5 } & Subtotal & 424 & 4.7 \\
\hline
\end{tabular}

* Deviation=[Accumulated flow rate per pump $-($ subtotal $\div 3)] \div($ subtotal $\div 3) \times 100$

\section{Conclusion}

The main goal of this study was to investigate the development of a software-based processor and experimental verification for detecting the flow rate of an individual pump in booster pump systems. In this study, integrated various functions without a flow sensor were developed and investigated in a smart booster pump system. The algorithm-based flow measurement method was developed to estimate the flow rate of each pump in the booster 
pump system. To verify the validity of the individual pump flow calculation processor, the average ensemble error was within $2 \%$ and the results for the experiment have been verified the accuracy of the development process. As a result of examining the accumulated flow rate for the sequential operation method and the optimum operation method, the accumulated flow rate of the specific pump was focused on the sequential operation method, whereas the optimal flow method distributes the accumulated flow evenly. By using the flow rate pattern, the deviation of each pump for the optimal operation was less than $\pm 5 \%$. Therefore, the algorithm-based flow estimation method was applied in a booster pump system and could be scaled in the industry.

Acknowledgements: The research work was supported by the Dooch Co., Ltd. For the promotion of science.

\section{References}

1. D. Kaya, E. A. Yagmur, K. S. Yigit, F. C. Kilic, A. S. Eren, and C. Celik, Energy efficiency in pumps, Energy Conversion and Management 49, 1662-1673 (2018)

2. R. Carlson, The correct method of calculating energy savings to justify adjustablefrequency drives on pumps, IEEE Transactions on Industry Applications 36(6), 1725 1733 (2000)

3. B. Schneider, Selection, operation and control of a work exchanger energy recovery system based on the Singapore project, Desalination 184(1), 197-210 (2005)

4. H. S. Ryu, Water Supply System Using Booster Pumps, Magazine of the SAREK 22(1), 1-16 (1993).

5. L. Jae-Hyun, P. Kwang-Ho, and H. Tae-Hoon, Life Cycle Cost Analysis of a General Booster Pump and High Efficiency Booster Pump, J. Architect. Inst. Korea Planning \& Design 22(9), 259-266 (2006)

6. J. K. Armintor, and D. P. Connors, Pumping Applications in the Petroleum and Chemical Industries, IEEE transactions on industry applications IA-23(1), 37-48 (1987)

7. M. Rakibuzzaman, Kyungwuk, H. -H. Kim, and S. -H. Suh, Energy saving rates for a multistage centrifugal pump with variable speed drive, J. of Power Technologies 97(2), 163-168 (2017)

8. Rakibuzzaman, S. -H. Suh, K. Kyungwuk, H. -H. Kim, M. T. Cho, and I. S. Yoon, A study on multistage centrifugal pump performance characteristics for variable speed drive system, Procedia Engineering 105, 270-275 (2015)

9. P.-A. Besse, G. Boero, M. Demierre, V. Pott, and R. Popovi, Detection of a single magnetic microbead using a miniaturized silicon Hall sensor, Applied Physics Letters 80(22), 4199-4201 (2002)

10. Jerry S. J. Chen, On the design of a wide range mini-flow paddlewheel flow sensor, Sensors and Actuators A: Physical 87(1-2), 1-10 (2000)

11. B. K. Bose, Modern power electronics and AC drives, Prentice-Hall, Inc., New Jersey, USA, 2002

12. R. W. Fox, A. T. Mcdonald, P. J. Pritchard, J. C. Leylegain, Fluid Mechanics, $8^{\text {th }}$ edition, John Wiley and Sons, Inc., (Asia), New Jersey, 2012

13. ISO 5198: 1987 (E), Centrifugal, mixed flow and axial pumps-code for hydraulic performance tests-precision class, International Standard. 